\title{
Comparison of Three-Dimensional Conformal Radiation Therapy, Intensity-Modulated Radiation Therapy, and Volu- metric-Modulated Arc Therapy In Glioblastoma Multiforme Radiation Therapy With EORTC Target Delineation
}

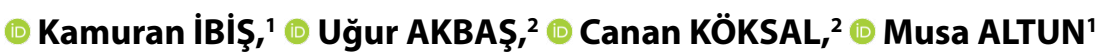 \\ 'Department of Radiation Oncology, Istanbul University, Institute of Oncology, Istanbul-Turkey \\ 2Department of Institute of Oncology, Istanbul University, Division of Medical Physics, Istanbul-Turkey
}

\begin{abstract}
OBJECTIVE
In this study, three-dimensional conformal radiation therapy (3D-CRT), intensity-modulated RT (IMRT), and volumetric-modulated arc therapy (VMAT) techniques were compared in patients with glioblastoma multiforme (GBM) receiving RT. Comparisons were made in terms of sparing the organs at risk (OAR), by using European Organisation for Research and Treatment of Cancer-Advisory Committee on Radiation Oncology Practise (EORTC-ACROP) guideline.
\end{abstract}

\section{METHODS}

RT in 10 patients was replanned. Treatment volume was created according to the EORTC-ACROP, and $60 \mathrm{~Gy} / 30$ fraction dose was prescribed for planning target volume (PTV). PTV-less brain volume (BPTV) Dmean, OARs doses; V5Gy and V50Gy of B-PTV volumes; conformality, and homogeneity indices were analyzed.

\section{RESULTS}

B-PTV was spared better in IMRT. The optic chiasm, contralateral optic nerve, ipsilateral/contralateral cochlea were significantly spared in IMRT and VMAT. The best sparing for brainstem, pituitary gland, ipsilateral eye, ipsilateral lacrimal gland was obtained with VMAT. B-PTV volume received at least 5 Gy was similar in three plans, but lower with $50 \mathrm{~Gy}$ in IMRT and VMAT ( $\mathrm{p}<0.001)$. Although homogenous dose distribution was obtained with similar homogeneity index in all three planning techniques, conformity index was the best in VMAT ( $<<0.001)$.

\section{CONCLUSION}

VMAT provides improved conformity index and good homogeneity in GBM RT using the EORTCACROP target and dose definition. The best sparing for OAR was obtained with VMAT.

Keywords: Glioblastoma multiforme; intensity-modulated radiotherapy; three-dimensional conformal radiotherapy; volumetric-modulated arc therapy.

Copyright $\odot$ 2018, Turkish Society for Radiation Oncology

\section{Introduction}

Concomitant and adjuvant temozolomide and radiation therapy (RT) are the standard treatment ap- proaches following maximal surgical resection for patients with glioblastoma multiforme (GBM).[1,2] Intensity-modulated radiation therapy (IMRT) is used as a treatment option, and it has been shown to provide 
similar or better target coverage and better preservation of normal tissues when compared to three-dimensional conformal RT (3D-CRT). [3-9] Studies comparing volumetric-modulated arc RT (VMAT) treatment planning with IMRT treatment plans have shown similar target coverage with better normal tissue sparing with VMAT (occasionally) and shorter treatment time. [8,10-13] In previous comparative studies for 3D-CRT and/or IMRT and/or VMAT preoperative and postopandor IMRT and erative MRI, only posto target volume margins, varying dose schedules (in-
cluding simultaneous integrated boost) have been used cluding simultaneous integrated boost) have

for target volume delineation. [6-9,12,14-19]
As emphasized in the guidelines for RT for glioblastoma published by the American Society for Radiation Oncology in March 2016, four main target delineations of different cooperative groups (single-phase treatment or two-phase treatment volume, involving or not involving edema) exist, and these are defined by the postperative MRI but have different target def itions. [2] We a and VMAT treatment plans on the doses of OARs, by using European Organisation for Research and Treatment of Cancer-Advisory Committee on Radiation Oncology Practise (EORTC-ACROP) target deline tion, which has not been evaluated previously. [20]

\section{Materials and Methods}

Ten consecutive patients who were diagnosed with GBM and underwent treatment were selected. Rigid fusion was performed using Mim-Version 6.5 (MIM Software Inc. US) program with the patients' simulation computed tomography (CT) imaging and postoperative magnetic resonance imaging (gadolinium contrast-enhanced T1-weighted MRI). Gross tumor volume (GTV) was defined as the surgical resection cavity plus any residual contrast-enhancing tumor (postcontrast T1-weighted MRI scans) as described in the ESTRO-ACROP guideline. Clinical target volume (CTV) was created by adding a 2 -cm margin to GTV. In addition, CTV was revised manually (tentorium, skull, falx cerebri, etc.) by considering the anatomical barrifalx cerebri, etc.) by considering the anatomical barri-
ers. The planning target volume (PTV) was created by ers. The planning target volume (PTV) was created by adding a margin of $5 \mathrm{~mm}$ to CTV. The ESTRO-ACROP guideline and the study of Scoccianti et al. were taken as a reference, and OARs and dose constraints were lineated by the same radiation oncologist. 3D-CRT MRT, and VMAT plans were generated on CT images for each patient by the same medical physicist. The prescribed dose to target was $60 \mathrm{~Gy} / 30$ fractions. The goal of the optimization for RT plans was to cover $95 \%$ target volume with $100 \%$ prescribed dose. The Eclipse treatment planning system (Varian, Palo Alto, CA) an Analytical Anisotropic Algorithm were used to create treatment plans. The calculation grid setting was $2.5 \mathrm{~mm}$, and 6-MV photon beams were utilized with Varian Trilogy linear accelerator. 3D-CRT plans were created using two opposing coplanar fields with a multileaf collimator margin of $5 \mathrm{~mm}$. I ated using seven coplanar fields separated with equa angles. Sliding window technique, which allows collimator leaves movement while irradiation continues, was chosen. Different collimator angles were used so as to provide high conformity and dose homogeneity. In this study, triple arc was performed for VMAT plans. The triple arc was consisted of three complete arcs set from $179^{\circ}$ to $181^{\circ}$ (counter clock wise), from $181^{\circ}$ to $179^{\circ}$ (clock wise) and from $179^{\circ}$ to $181^{\circ}$ (counter clock ise), ( the collimator angles were defined as $30^{\circ}, 330^{\circ}$, and $90^{\circ}$ for all VMAT plans. The reason of choosing differen collimator angles was to avoid the tongue-and-groove effect. For a fair comparison, the same optimization template was used with IMRT. Reoptimizations were made until the desired results were acquired for both IMRT and VMAT planning.

PTV volume was removed from normal brain tissue PTV volume was removed fron and brain-PTV (B-PTV) volume was generated. Dose constraints for OARs: optic chiasm maximum dose (Dmax) <54 Gy, (secondary criteria: Dmax <60 Gy), optic nerve Dmax <54 Gy (secondary criteria: $55 \mathrm{~Gy}$ ), cochlear mean dose (Dmean) $<45$ Gy, brainstem Dmax $<54$ Gy (secondary criteria: Dmax <60 Gy, D59Gy <10 $\mathrm{ml}$ ), pituitary gland Dmax $<50 \mathrm{~Gy}$ (secondary criteria Dmax $<60 \mathrm{~Gy}$ ), the eyes Dmax $<45 \mathrm{~Gy}$, lacrimal gland Dmax $<40 \mathrm{~Gy}$ intraocular lens Dmax $<6 \mathrm{GY}$ (secondary criteria: $<10 \mathrm{~Gy}$; Table 1).

Table 1 Organs at risk and dose constraints

\begin{tabular}{|c|c|c|}
\hline Organs at risk & Constraints & Secondary criteria \\
\hline Optic chiasma & Dmax<54 Gy & Dmax $<60$ Gy \\
\hline Optic nerve & Dmax<54 Gy & Dmax<55 Gy \\
\hline Cochlea & Dmean<45 Gy & \\
\hline Brainstem & Dmax<54 Gy & Dmax $<60$ Gy, \\
\hline D59 Gy<10 cc & & \\
\hline Pituitary gland & Dmax<50 Gy & Dmax $<60$ Gy \\
\hline Eye & Dmax $<45$ Gy & \\
\hline Lacrimal gland & Dmax<40 Gy & \\
\hline Lens & Dmax<6 Gy & Dmax $<10$ Gy \\
\hline
\end{tabular}

Conformity index and homogeneity index were calculated for all treatment plans. Conformity index was defined as the ratio between the tissue volume included in the reference isodose ( $95 \%$ prescribed dose) and the PTV volume (ml; ICRU 62; conformity index=Vri/ PTV). The optimal conformity index was 1.[22] The homogeneity index was calculated to evaluate the homogeneity of the dose distribution within the PTV. It is defined as the ratio of the difference between dose 2\% (almost mum) of the target and the median dose to the target homogeneity index $=(\mathrm{D} 2 \%-\mathrm{D} 98 \%) / \mathrm{D} 50 \%$ (ICRU 83). Doses of B-PTV Dmean, V5Gy, and V50Gy of B-PTV volumes, optic chiasm Dmax, ipsilateral/contralateral optic nerve Dmax and cochlear Dmean, brainstem Dmax, pituitary gland Dmax, ipsilateral/contralateral eye Dmax, ipsilateral/contralateral lacrimal gland Dmax, ipsilateral/contralateral intraocular lens Dmax; and conformity and homogeneity index were statistically compared by paired sample t-test. A P v $<0.05$ was considered statistically significant.

\section{Results}

Median PTV was $303.6 \mathrm{ml}$ (151-502 ml). Tumor localization was located in right parietal lobe in one patient, right temporoparietal lobe in one patient, right temporal lobe in four patients, right temporo-occipital lobe in one patient, left frontal lobe in two patients, and left temporal lobe in one patient. The lowest (median (1.5 Gy) B-PTV D oedian In the VMAT plan, the median dose of B-PTV Dmean was 2 Gy higher than IMRT plan. The median dose of 3D-CRT plan was 10.8 Gy higher than the IMRT plan (Table 2). B-PTV volume that received at least 5 Gy was similar in three plans, but it is significantly lower with 50 Gy in IMRT (8.9\%) and VMAT (8.7\%) than 3DCRT (44.1\%; p $<0.001$; Fig. 1).

Optic chiasm Dmax median doses of 60.6 Gy, 50.1 Gy, 51 Gy were detected in 3D-CRT, IMRT, VMAT treatment plans, respectively. Optic chiasm was significantly better preserved in IMRT and VMAT planning methods (3D-CRT vs. IMRT, $p=0.021$; 3D-CRT ning methods $(3 \mathrm{D}$-CRT vs. IMRT, $\mathrm{p}=0.021$; $3 \mathrm{D}$-CRT
vs. VMAT, $\mathrm{p}=0.008)$. There was no difference between vs. VMAT, $\mathrm{p}=0.008)$. There was no differe
IMRT and VMAT ( $\mathrm{p}=0.205$; Table 2 ).

The contralateral optic nerve was significantly bet-
The and VMAT $(\mathrm{p}=0.205$; Table 2 . ter preserved in both IMRT (median 22.4 Gy) and VMAT (median 27.2 Gy) when compared to 3D-CRT (median $61.5 \mathrm{~Gy}$ ). There was no statistically significant difference between IMRT and VMAT. Although there was no statistically significant difference between the

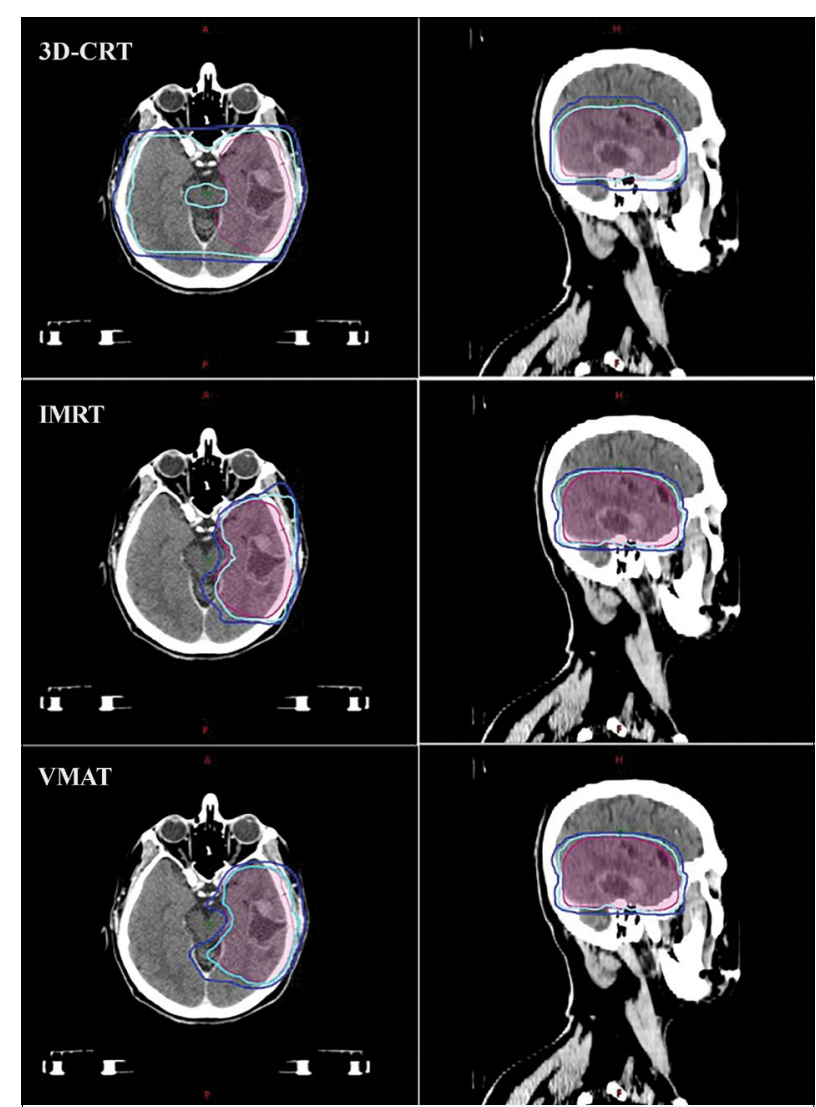

Fig. 1. Axial and sagittal images of 50-Gy and 60-Gy isodose lines in three different plannings.

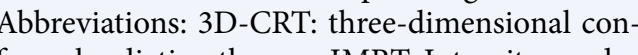
ford The blue line: 50-Gy iodose line:

three plans at the median dose of the optic nerve, it was better preserved with IMRT and VMAT (Table 2).

Brainstem Dmax median doses of $60.3 \mathrm{~Gy}, 54.3 \mathrm{~Gy}$, 52.8 Gy were detected in 3D-CRT, IMRT, and VMAT treatment plans, respectively. The brainstem was significantly better preserved in IMRT and VMAT (3DCRT vs. IMRT, $\mathrm{p}<0.001 ; 3 \mathrm{D}-\mathrm{CRT}$ vs. VMAT, $\mathrm{p}=0.015$ ). VMAT preserved brainstem better than IMRT $(\mathrm{p}=0.015$; Table 2).

Pituitary gland Dmax median doses were $60.1 \mathrm{~Gy}$ 47.7 Gy, and 42.7 Gy in 3D-CRT, IMRT, VMAT, respectively. Pituitary gland sparing was statistically better in IMRT and VMAT than in 3D-CRT, whereas the lowest organ dose was obtained in VMAT ( $\mathrm{p}=0.009, \mathrm{p}=0.003$, $\mathrm{p}=0.023$, respectively; Table 2 )

The contralateral cochlea and ipsilateral cochlea doses were significantly higher in IMRT (median 13.4 Gy and $22.6 \mathrm{~Gy}$ respectively) and VMAT (median 14 
Table 2 Comparison of distribution of doses on normal tissues of EORTC volume planning through three different techniques in glioblastoma multiforme radiation therapy

\begin{tabular}{|c|c|c|c|c|c|c|}
\hline & $\begin{array}{c}\text { A } \\
\text { X } \pm \text { SD Median } \\
\text { (min-max) }\end{array}$ & $\begin{array}{c}\text { B } \\
\text { X } \pm \text { SD Median } \\
\text { (min-max) }\end{array}$ & $\begin{array}{c}\text { C } \\
\text { X } \pm \text { SD Median } \\
(\text { min-max })\end{array}$ & $\begin{array}{c}\text { A-B } \\
\text { p value }\end{array}$ & $\begin{array}{c}\text { A-C } \\
\text { p value }\end{array}$ & $\begin{array}{c}\text { B-C } \\
\text { p value }\end{array}$ \\
\hline Brain-PTV & $34 \pm 10.3$ & $21.6 \pm 4.3$ & $23.8 \pm 5.2$ & & & \\
\hline \multirow[t]{2}{*}{ Dmean } & 32.3 & 21.5 & 23.5 & $<0.001$ & 0.001 & 0.008 \\
\hline & $(15.2-50.5)$ & $(16.5-30.5)$ & $(14.9-33.3)$ & & & \\
\hline \multirow[t]{2}{*}{ Optic chiasm Dmax } & $53.5 \pm 18.7$ & $44 \pm 11.8$ & $41.7 \pm 16.7$ & & & \\
\hline & $\begin{array}{c}60.6 \\
(1-62.2)\end{array}$ & $\begin{array}{c}50.1 \\
(18.8-54.9)\end{array}$ & $\begin{array}{c}51 \\
(8.5-52.2)\end{array}$ & 0.021 & 0.008 & 0.205 \\
\hline \multirow[t]{2}{*}{ Contralateral optic nerve } & $44.25 \pm 28.7$ & $22.7 \pm 11$ & $23.9 \pm 11.5$ & & & \\
\hline & 61.5 & 22.4 & 27.2 & 0.011 & 0.007 & 0.426 \\
\hline \multirow{2}{*}{ Ipsilateral optic nerve } & $\begin{array}{l}(0.53-63.1) \\
44.16+28.8\end{array}$ & $\begin{array}{l}(1.1-45.5) \\
393+156\end{array}$ & $\begin{array}{l}(6.3-39) \\
373+192\end{array}$ & & & \\
\hline & $\begin{array}{c}61.3 \\
(0.5-63.4)\end{array}$ & $\begin{array}{c}48.7 \\
(12-53.6)\end{array}$ & $\begin{array}{c}49.3 \\
(7.3-52.2)\end{array}$ & 0.327 & 0.068 & 0.210 \\
\hline \multirow[t]{2}{*}{ Contralateral cochlea Dmean } & $36.7 \pm 25.7$ & $11.9 \pm 7.8$ & $10.9 \pm 7.1$ & & & \\
\hline & $\begin{array}{c}53.8 \\
(0.7-58.3)\end{array}$ & $\begin{array}{c}13.4 \\
(1.1-21.7)\end{array}$ & $\begin{array}{c}14 \\
(0.9-19.1)\end{array}$ & 0.003 & 0.003 & 0.303 \\
\hline \multirow[t]{2}{*}{ Ipsilateral cochlea Dmean } & $41.5 \pm 27.4$ & $19.9 \pm 14$ & $20 \pm 12.7$ & & & \\
\hline & $\begin{array}{c}58.7 \\
(0.6-60.3)\end{array}$ & $\begin{array}{c}22.6 \\
(1.1-43)\end{array}$ & $\begin{array}{c}26.2 \\
(1.2-33.3)\end{array}$ & 0.002 & 0.002 & 0.974 \\
\hline \multirow{2}{*}{ Brainstem Dmax } & $54.6 \pm 18$ & $50 \pm 10.3$ & $47.2 \pm 12.9$ & & & \\
\hline & 60.3 & 54.3 & 52.9 & 0.147 & 0.015 & 0.015 \\
\hline Pituiter gland & $\begin{array}{l}(3.9-66.6) \\
46.9 \pm 22.3\end{array}$ & $\begin{array}{c}(23.5-55.9) \\
38 \pm 17.1\end{array}$ & $\begin{array}{l}(114 .-155.7) \\
34.5 \pm 17.8\end{array}$ & & & \\
\hline \multirow[t]{2}{*}{ Dmax } & 60.1 & 47.7 & 42.7 & 0.009 & 0.001 & 0.049 \\
\hline & $(0.8-61.5)$ & $(6.1-50.6)$ & $(5-49.2)$ & & & \\
\hline \multirow[t]{3}{*}{ Contralateral eye Dmax } & $28.8 \pm 28.2$ & $19.9 \pm 13.2$ & $18.8 \pm 8.8$ & & & \\
\hline & 20.1 & 13.9 & 16.2 & 0.130 & 0.155 & 0.473 \\
\hline & $(0.3-62.9)$ & $(5.6-41.6)$ & $(6.8-32.3)$ & & & \\
\hline \multirow{3}{*}{ Ipsilateral eye Dmax } & $30.8 \pm 30.2$ & $36.3 \pm 9.9$ & $28.2 \pm 15.3$ & & & \\
\hline & 22.9 & 34.5 & 24 & 0.454 & 0.612 & 0.006 \\
\hline & $(0.4-65.6)$ & $(21.5-52.9)$ & $(8.9-47.1)$ & & & \\
\hline Contralateral lacrimal & $27.3 \pm 27.5$ & $16 \pm 9.2$ & $18.1 \pm 9.3$ & & & \\
\hline \multirow{2}{*}{ gland Dmax } & 17.7 & 12.2 & 16.4 & 0.103 & 0.173 & 0.131 \\
\hline & $(0.3-62.7)$ & $(5.7-33.7)$ & $(6.8-34.7)$ & & & \\
\hline Ipsilateral lacrimal gland & $31.5 \pm 31.3$ & $33.5 \pm 9.6$ & $26.7 \pm 12.5$ & & & \\
\hline \multirow[t]{2}{*}{ Dmax } & 27.7 & 36.2 & 27.8 & 0.797 & 0.456 & 0.002 \\
\hline & $(0.3-65.9)$ & $(15.2-43.6)$ & $(10.3-40.5)$ & & & \\
\hline \multirow[t]{3}{*}{ Contralateral lens Dmax } & $5.7 \pm 5.7$ & $5.9 \pm 1$ & $6.2 \pm 2$ & & & \\
\hline & 3.1 & 6.2 & 6 & 0.917 & 0.719 & 0.391 \\
\hline & $(0.2-16.6)$ & $(3.9-7.35)$ & $(4-10.5)$ & & & \\
\hline \multirow{3}{*}{ Ipsilateral lens Dmax } & $4.1 \pm 4.1$ & $7.4 \pm 1.9$ & $6.8 \pm 2.7$ & & & \\
\hline & 2.1 & 7.2 & 6.5 & 0.008 & 0.019 & 0.079 \\
\hline & $(0.2-12)$ & $(4.4-10.5)$ & $(3.1-12.4)$ & & & \\
\hline \multirow[t]{3}{*}{ Homogeneity index } & $0.07 \pm 0.03$ & $0.06 \pm 0.02$ & $0.06 \pm 0.01$ & & & \\
\hline & 0.07 & 0.07 & 0.06 & 0.601 & 0.256 & 0.078 \\
\hline & $(0.01-0.12)$ & $(0.03-0.09)$ & $(0.04-0.09)$ & & & \\
\hline \multirow{3}{*}{ Conformity index } & $2.2 \pm 0.3$ & $1.2 \pm 0.03$ & $1 \pm 0.02$ & & & \\
\hline & 2.3 & 1.1 & 1 & $<0.001$ & $<0.001$ & $<0.001$ \\
\hline & $(1.8-2.4)$ & $(1.1-1.2)$ & $(1-1.06)$ & & & \\
\hline
\end{tabular}

A: 3 dimensional conforma
Planning target volume
Gy and $26.2 \mathrm{~Gy}$, respectively) than in 3D-CRT (median $53.8 \mathrm{~Gy}$ and $58.7 \mathrm{~Gy}$, respectively; $\mathrm{p}=0.003$ and $\mathrm{p}=0.002$, respectively). Bilateral cochlear sparing was similar in IMRT and VMAT (Table 2).

The contralateral eye was similarly spared in all three plans. There was no statistical difference between the ipsilateral eye Dmax in 3D-CRT (median 22.9 Gy, 0.4-65.6), IMRT (median 34.5 Gy, 21.5-52.9) and VMAT (median 24 Gy; 8.9-47.1). However, the ipsilateral eye Dmax was statistically lower in VMAT than in IMRT $(\mathrm{p}=0.006)$. VMAT provides the best sparing for ipsilateral eye (Table 2).

The contralateral lacrimal gland was similarly spared in all three plans. There was no statistical difference for the ipsilateral lacrimal gland Dmax between 3D-CRT (median 27.7 Gy, 0.3-65.9), IMRT (median 36.2 Gy, 15.2-43.6), and VMAT (median $27.8 \mathrm{~Gy}$, 10.3-40.5). However, ipsilateral lacrimal Dmax significantly lower in VMAT than IMRT ( $\mathrm{p}=0.002)$. VMAT showed the best sparing for the ipsilateral lacrimal gland (Table 2)

Median doses of contralateral intraocular lens Dmax were 3.1 Gy, 6.2 Gy, 6 Gy in 3D-CRT, IMRT, and VMAT treatment plans, respectively; there were no significant difference between three plans. Median doses of ipsilateral intraocular lens Dmax were 2.1 Gy, 7.2 Gy, 6.5 Gy in 3D-CRT, IMRT, and VMAT treatment plans, respectively. There was no significant difference between IMRT and VMAT, but the lowest dose was obtained with 3D-CRT (Table 2).

A similar homogeneity index was obtained in all three plans. Conformity index median values were $2.3,1.1$, and 1 in 3D-CRT, IMRT, and VMAT treatment plans, respectively. Compared with 3D-CRT, a more conformal treatment plan was obtained with both IMRT and VMAT. Although the difference was low, VMAT treatment plan was statistically significant more conformal (Table 2).

\section{Discussion}

Some studies comparing different treatment planning techniques have been published in the literature. There are six studies comparing 3D-CRT and IMRT. $[3,4,6,7,18,23]$ Lorentini et al. replanned 17 GBM patients previously treated with both 3D-CRT and IMRT. [18] They described GTV as the resection cavity plus any contrast-enhancing area in post-gadolinium T1weighted MRI. CTV was created by adding 2-cm margin to GTV. Natural anatomical barriers (e. bone tentorium, falx) were then manually corrected. CTV was established by adding a $0.5-\mathrm{cm}$ margin to PTV. They have reported that IMRT provides better target coverage while providing similar OARs sparing and decreased healthy brain irradiation. Thibouw et al. studied retrospectively 220 patients with glioblastoma treated with 3D-CRT and IMRT.[23] They compared dosimetric parameters as well as clinical and survival data with the aid of these two techniques. As a result they reported thatbetter targetconformity was achieved in patients by IMRT, and a reduction in neuroged in CRT CRT and IMRT plans in 20 patients with high-grade glioma. GTV, in T1-weighted MRI scan, was defined a the post-resection cavity plus any residual contrast-en hancing tissue.[6] CTV was created by adding a $2-\mathrm{cm}$ margin to GTV. PTVinitial was created by adding a $0.5-\mathrm{cm}$ margin to CTV, and PTVboost was created by adding a $0.5-\mathrm{cm}$ margin to GTV; hence, a two-phase treatment plan was formed. As a result, they reported that IMRT provides improved target conformity and better sparing for OARs without increasing the better spaing for OARs withoul increasing the volune of nomal tisste irradiated with integral dose and low-dose radiation. The brainstem, optic chiasm, bilateral optic nerves were reported to receive lower doses. MacDonald et al. dosimetrically compared 3D-CRT with IMRT plan in 20 patients with high-grade glioma. [3] The prescribed dose was 59.4 Gy delivered at 1.8 Gy per fraction. Eventually, they reported that IMRT improved target coverage and decreased radiation dose in the brain, brainstem, and optic chiasm. Chan et al. applied 59.4 Gy for GTV lus a margin of $2.5 \mathrm{~cm}$ in D 70 Gy for GTV in IMRT with a sin in IMRT.[4] They showed that IMRT better preserved normal brain and other critical structures, and that it could be applied simultaneous boost for GTV. Piroth et al. compared 3D-CRT and integrated-boost IMRT by using preoperative and postoperative MRI and in cluding perifocal edema around the tumor.[7] The total dose was prescribed for $72 \mathrm{~Gy}$ and $60 \mathrm{~Gy}$ for PTV and PTV2, using daily fractions of 2.4 and $2 \mathrm{~Gy}$. They and They achieved more homogeneity and conformity with integrated-boost IMRT.In our study, we performed a targe 政 three treatment plans, total radiation dose was defined as $60 \mathrm{~Gy}$ in 30 fractions in a single phase. Compared to IMRT and VMAT, B-PTV Dmean dose was the highest in 3D-CRT. Optic chiasm Dmax, contralateral optic nerve and bilateral cochlear Dmean, pituitary gland Dmax, ipsilateral intraocular lens Dmax were significantly higher in 3D-CRT. Conformity index w 
tistically significant lower, although 3D-CRT showed similar homogeneity to the other two treatment plans. Buglione et al. compared 3D-CRT, IMRT, and tomotherapy and defined GTV as areas that contrasted with preoperative/postoperative MRI T1 sequences in 10 patients with glioblastoma. CTV was defined as GTV plus a 2-cm margin.[14] They modified CTV according to anatomical boundaries such as skull bones, ventricles, and OARs. The prescribed dose was $60 \mathrm{G}$. They reported a significant dosimetric advantage with the therey in comparison to 3D-CRT and IMRT. Anomotherapy in compris similar study was published by Zach et al. [9] They constructed four different treatment plans, including $3 \mathrm{D}$ CRT, sequential boost IMRT, integrated-boost IMRT, and tomotherapy, by using two-phase dose definition for 20 high-grade glioma patients. Peritumoral edema was included when defining the treatment volume. At the end of the study, optic chiasm, and ipsilateral glob mean doses were the highest in the 3D-CRT plan, whereas the lowest in integrated-boost IMRT. Con lateral glob mean dose was the highest in tomotherapy plan. The mean of the integral dose to the brain was least with the integrated-boost plan and was lower with IMRT than in 3D-CRT. The researchers reported that the single treatment planning method was not superior to the others. In the present study, the best B-PTV mean dose was achieved with the IMRT plan.

Adeberg et al. compared IM proton therapy (PRT), VMAT, and 3D-CRT treatment plans in 12 patients with high grade glial tumors. [15] They used a volume definition containing tumor cavity and edema in postoperative MRI. Compared with 3D-CRT and VMAT, PRT showed a statistically significant dose reduction in whole-brain mean dose, brainstem, pituitary gland, contralateral hippocampus, and contralateral subventricular zone.

Navarria et al. performed treatment plan assessment, progression-free survival, and overall survival analysis in patients with high-grade 341 gliomas treated with 3D-CRT and VMAT [17] They created CTV by adding DD-CRT an is $10-\mathrm{mm}$. 17 arg in to GTV on preopeding an isotreic 10-mive contry on postoper Tl-weight, and the resection cavity on postoperative MRI, and the presence of abnormality FLAIR area, and if present, the residual tumor. They defined PTV by expanding CTV isotropic by $3 \mathrm{~mm}$. They reported that VMAT is superior to 3DCRT in dosimetric and clinical results.

In the first of three studies comparing VMAT and IMRT, Shaffer et al. evaluated these two planning methods dosimetrically in 10 patients with frontal and temporal high-grade glioma.[12] They defined GTV as contrast-enhancing tumor volume on T1-weighted MRI scans. GTV was expanded by $2 \mathrm{~cm}$; hence, CTV was formed after incorporating postoperative tumo area and T2-weighted MRI (three-dimensionally). CTV was expanded by $0.5 \mathrm{~cm}$ to create PTV. They used single-phase plan with $60 \mathrm{~Gy}$ in 30 fractions. As a result of the study, PTV coverage, homogeneity, and conformity were found to be similar/equal. They reported statistically significant decrease in VMAT maximum and mean retinal, intraocular lens, and contralatera optic nerve doses. Davidson et al. compared IMRT single-arc VMAT, and the addition of partial arc plan in six brainstem gliomas and six GBM patients.[16] In patients with GBM, GTV was defined as the postoperative tumor volume in post-gadolinium enhancing $\mathrm{T} 1$ weighted MRI scan. CTV was created by adding $1.5 \mathrm{~cm}$ margin to GTV, and PTV was created by adding 0.5 $\mathrm{cm}$ margin to CTV. It has been reported that VMAT provided similar dosimetric quality to IMRT but provided faster treatment delivery. Briere et al. compared VMAT and IMRT in 90 patients with GBM in whom 50 Gy was administered in 30 fractions for PTV and $60 \mathrm{~Gy}$ was administered in 30 fractions for simultaneous integrated-boost PTV.[19] They defined GTV and CTV according to the ESTRO-ACROP guideline but differently applied simultaneous integrated boost. Mean dose in the brainstem, ipsilateral, and contralateral cochlea was lower in VMAT. Total treatment time was 5 min shorter. Compared VMAT to IMRT in patients with GBM, the similar target coverage, better sparing of brainstem and cochlea, and shorte duration of treatment can be achieved. In the presen study, maximal doses of the brainstem, pituitary gland, ipsilateral eye, and ipsilateral lacrimal gland were significantly lower with VMAT compared to IMRT, and better OARs sparing were obtained.

Wagner et al. compared VMAT, IMRT, and 3D-CRT, which is only such study in the literature.[8] GTV in 14 patients defined as primary tumor/tumor field in T1-weighted preoperative MR images. CTV was created by expanding the GTV in all directions by $1.5 \mathrm{~cm}$. IMRT technique showed better PTV coverage than VMAT. The advantage of VMAT is shorter treatment time, lower monitor units, and a small V107\%. If the PTV is distant from the OAR, use of the 3D-CRT technique is safe. In other cases, the intensity-modulated technique should be used. In the present study, the target coverage with IMRT and VMAT were statistically significant better than 3D-CRT

\section{Conclusion}

Normal brain tissue was best spared in the IMRT plan among three different treatment plans assessed, according to EORTC-ACROP guide target volume and treatment dose definition. The maximum doses of the contralateral eye, lacrimal gland, and intraocular lens were similar in all three plans. Although the maximum doses of optic chiasm, ipsilateral intraocular lens and, the mean doses of bilateral cochlea were lower in IMRT and VMAT than in 3D-CRT, both methods provided similar preservation. The maximum doses of ipsilatera eye and lacrimal ghand were similar in 3D-CRT and eye and hcrim gher The maximum doses of brainstem and pituitary gland were lower in VMAT than in the other planning methods. VMAT provided improved conformity index and good homogeneity in GBM RT using the EORTCACROP target and dose definition. The best sparing for OARs was obtained with VMAT technique.

Peer-review: Externally peer-reviewed.

Conflict of Interest: The authors declare that there is no conflict of interest.

Authorship contributions: Concept - K.I.j; Design - K.I. Supervision - K.I.,, M.A.; Materials - K.I., M.A. Data collection \&/or processing - KJ. U.A., C.K. Analysis and/or interpretation - K.I.., U.A., M.A.; Literature search - K.I.I. C.K. Writing - K.İ., M.A.; Critical review - K.İ., M.A.

\section{References}

1. Stupp R, Hegi ME, Mason WP, van den Bent MJ, Taphoorn M), anzer RC, et al; European Organisation for Research and Treatment of Cancer Brain Tumour and Radiation Oncology Groups; National Cancer Institute of Canada Clinical Trials Group. Effects of radiotherapy with concomitant and adjuvant temozolomide versus radiotherapy alone on survival in glioblastoma in a randomised phase III study: 5-year analysis of the EORTC-NCIC trial. Lancet Oncol 2009;10(5):459-66.

2. Cabrera AR, Kirkpatrick JP, Fiveash JB, Shih HA, Koay EJ, Lutz S, et al. Radiation therapy for glioblastoma: Executive summary of an American Society for Radiation Oncology Evidence-Based Clinical Practice diation Oncology Evidence-Based Clinical Practictine. Pract Radiat Oncol 2016;6(4):217-25.

3. MacDonald SM, Ahmad S, Kachris S, Vogds BJ, DeRouen $M$, Gittleman $A E$, diation therapy versus three-dimens modulated raradiation therapy for the treatment of high grade glia 2007;8(2):47-60.
4. Chan MF, Schupak K, Burman C, Chui CS, Ling CC. Comparison of intensity-modulated radiotherapy with three-dimensional conformal radiation therapy planning for glioblastoma multiforme. Med Dosim 2003;28(4):261-5.

5. Narayana A, Yamada J, Berry S, Shah P, Hunt M, Gutin $\mathrm{PH}$, et al. Intensity-modulated radiotherapy in highgrade gliomas: clinical and dosimetric results. Int Radiat Oncol Biol Phys 2006;64(3):892-7.

6. Hermanto U, Frija EK, Lii MJ, Chang EL, Mahajan A, Woo SY. Intensity-modulated radiotherapy (IMRT) and conventional three-dimensional conformal radiotherapy for high-grade gliomas: does IMRT increase the integral dose to normal brain? Int J Radiat Oncol Biol Phys 2007;67(4):1135-44.

7. Piroth MD, Pinkawa M, Holy R, Stoffels G, Demirel C, Attieh C, et al. Integrated-boost IMRT or 3-D-CRT using FET-PET based auto-contoured target volume delineation for glioblastoma multiforme-a dosimetric comparison. Radiat Oncol 2009;4:57.

8. Wagner D, Christiansen H, Wolff H, Vorwerk $\mathrm{H}$. Radiotherapy of malignant gliomas: comparison of volumetric single arc technique (RapidArc), dynamic volumetric single arc technique (RapidArc), dynamic technique. Radiother Oncol 2009;93(3):593-6.

9. Zach L, Stall B, Ning H, Ondos I, Arora B, Uma S, et al. A dosimetric comparison of four treatment planning methods for high grade glioma. Radiat Oncol 2009;4:45.

10. Amelio D, Lorentini S, Schwarz M, Amichetti M Intensity-modulated radiation therapy in newly diagnosed glioblastoma. a systematic review on clinica and technical issues.

11.Cao D, Holmes TW, Afghan MK, Shepard DM. Comparison of plan quality provided by intensity-modulated arc therapy and helical tomotherapy. Int J Radia Oncol Biol Phys 2007;69(1):240-50.

12. Shaffer R, Nichol AM, Vollans E, Fong M, Nakano S, Moiseenko V, et al. A comparison of volumetric modulated arc therapy and conventional intensitymodulated radiotherapy for frontal and tempora high-grade gliomas. Int J Radiat Oncol Biol Phys 2010;76(4):1177-84.

13. Panet-Raymond V, Ansbacher W, Zavgorodni S, Bendorffe B, Nichol A, Truong PT, et al. Coplanar versus noncoplanar intensity-modulated radiation therapy (IMRT) and volumetric-modulated arc therapy (VMAT) treatment planning for fronto-temporal highgrade glioma. J Appl Clin Med Phys 2012;13(4):3826. guglione M Spiazzi L Saini F Costa L Sh):3826. Buglione M, Spiazzi L, Saiani F, Costa L, Shehi B, Lazzari B, et al; Neuro-Oncology Group, Spedali Civili conformal radiotherapy, static intensity-modulated 
and helical intensity-modulated radiotherapy in glioblastoma. Dosimetric comparison in patients with overlap between target volumes and organs at risk. Tumori 2014;100(3):272-7.

15. Adeberg S, Harrabi SB, Bougatf N, Bernhardt D, Rieber J, Koerber SA, et al. Intensity-modulated proton therapy, volumetric-modulated arc therapy, and 3D conformal radiotherapy in anaplastic astrocytoma and glioblastoma : A dosimetric comparison. Strahlenther Onkol 2016;192(11):770-9.

16. Davidson MT, Masucci GL, Follwell M, Blake SJ, Xu W, Moseley DJ, et al. Single arc volumetric modulated arc therapy for complex brain gliomas: is there an advantage as compared to intensity modulated radiotherapy or by adding a partial arc? Technol Cancer Res Treat 2012;11(3):211-20.

17. Navarria P, Pessina F, Cozzi L, Ascolese AM, Lobefalo F, Stravato A, et al. Can advanced new radiation therapy technologies improve outcome of high grade glioma (HGG) patients? analysis of 3D-conformal radiotherapy (3DCRT) versus volumetric-modulated arc therapy (VMAT) in patients treated with surgery, concomitant and adjuvant chemo-radiotherapy. BMC Cancer 2016;16:362.

18. Lorentini S, Amelio D, Giri MG, Fellin F, Meliado G,
Rizzotti A, et al. IMRT or 3D-CRT in glioblastoma? A dosimetric criterion for patient selection. Technol Cancer Res Treat 2013;12(5):411-20.

19. Briere TM, McAleer MF, Levy LB, Yang JN. Sparing of normal tissues with volumetric arc radiation therapy for glioblastoma: single institution clinical experience. Radiat Oncol 2017;12(1):79.

20. Niyazi M, Brada M, Chalmers AJ, Combs SE, Erridge SC, Fiorentino A, et al. ESTRO-ACROP guideline "target delineation of glioblastomas". Radiother Oncol 2016;118(1):35-42.

21. Scoccianti S, Detti B, Gadda D, Greto D, Furfaro I, Meacci F, et al. Organs at risk in the brain and their dose-constraints in adults and in children: a radiation oncologist's guide for delineation in everyday practice. Radiother Oncol 2015;114(2):230-8.

22. Feuvret L, Noël G, Mazeron JJ, Bey P. Conformity index: a review. Int J Radiat Oncol Biol Phys 2006;64(2):333-42.

23. Thibouw D, Truc G, Bertaut A, Chevalier C, Aubignac L, Mirjolet C. Clinical and dosimetric study of radiotherapy for glioblastoma: three-dimensional conformal radiotherapy versus intensity-modulated radiotherapy. J Neurooncol 2018;137(2):429-38. 